\title{
Unusual presentation of hyperhomocysteinaemia
}

\author{
Authors: Deepak Ramnani, ${ }^{\mathrm{A}}$ Swati Kapoor ${ }^{\mathrm{A}}$ and Rajeev Upreti ${ }^{\mathrm{B}}$
}

\section{Introduction}

Acute portomesenteric vein thrombosis is an uncommon but serious condition with potential sequelae, such as small bowel gangrene and end-stage hepatic failure. Here we describe a rare association of hyperhomocysteinaemia with isolated portomesentric thrombosis.

\section{Case}

A 39-year-old gentleman presented with a complaint of severe diffuse abdominal pain along with episodes of non-projectile non-bloody vomiting. The patient also had history of recurrent episodes of diffuse abdominal pain since a few days ago which were not as severe in intensity and were relieved by oral analgesics. This episode was not associated with any change in frequency of stools, fever or any history of travel or eating out. The patient was a vegetarian, non-smoker and was a social drinker with no alcohol intake in recent times. On examination, the patient was dehydrated, hypotensive and had a pulse rate of 110 beats $/ \mathrm{min}$. There was guarding of the abdomen with diffuse tenderness and bowel sounds were sluggish. An X-ray was immediately done which was not suggestive of any obstruction. His laboratory tests however revealed low haemoglobin, with a macrocytic picture on peripheral smear. Meanwhile computed tomography of the whole abdomen with angiography revealed superior mesenteric vein thrombosis, portal vein thrombosis along with jejunal wall ischaemia and moderate free fluid. In view of that the vascular surgery team was consulted and anticoagulant (unfractionated heparin) was started under close monitoring.

\section{Results and discussion}

His coagulation profile revealed a high homocysteine level $(45.8 \mu \mathrm{mol} / \mathrm{L})$, while the rest of the parameters (activated partial thromboplastin time, international normalising ratio, lupus anticoagulant factor V, protein S and C, JAK 2 mutation) were within normal limits. Partial thromboplastin time was slightly above the normal range.

In view of high homocysteine levels and macrocytosis, the patient's vitamin $B_{12}$ levels were sent to laboratory and they turned out to be low.

Authors: ${ }^{A}$ Max Super Speciality Hospital, Saket, Delhi, India;

${ }^{B}$ George Eliot Hospital, Nuneaton, Warwickshire, UK
In the urgent exploratory laparotomy that was performed, a $30 \mathrm{~cm}$ gangrenous portion of small bowel was resected and anastomosis was done using stapler. The patient's postoperative period was uneventful and he was discharged after 5 days in stable condition. The patient was supplemented with anticoagulants and vitamin $B_{12}$ on discharge. Thus, this is a rare case of hyperhomocysteinaemia presenting with portomesentric thrombosis.

Hyperhomocysteinaemia has been well documented to be associated with increased risk of arterial thrombotic events, ${ }^{1}$ peripheral arterial disease ${ }^{2}$ and stroke. ${ }^{3}$ It is also a risk factor for deep-vein thrombosis in the general population. ${ }^{4,5}$ However, its association with portal vein thrombosis is very unusual and only a few cases have been reported.

\section{Conclusion}

Portomesentric vein thrombosis is in itself a rare cause of abdominal pain, and in the absence of hepatic disease, an underlying thrombophilia should be suspected. We emphasise the importance of measuring serum homocysteine in all patients with portal vein thrombosis to avoid missing this rare but curable condition. Management of acute portal vein thrombosis is complex because of the lack of evidence-based therapeutic algorithms. Close cooperation between surgery, radiology and internal medicine is crucial.

\section{Conflict of interest statement}

None declared.

\section{References}

1 Stampfer MJ, Malinow MR, Willett WC et al. A prospective study of plasma homocyst(e)ine and risk of myocardial infarction in US physicians. JAMA 1992;268:877-81.

2 Khandanpour N, Loke YK, Meyer FJ, Jennings B, Armon MP. Homocysteine and peripheral arterial disease: systematic review and meta-analysis. Eur J Vasc Endovasc Surg 2009;38:316-22.

3 Towfighi A, Markovic D, Ovbiagele B. Pronounced association of elevated serum homocysteine with stroke in subgroups of individuals: a nationwide study. J Neurol Sci 2010;298:153-7.

4 Köktürk N, Kanbay A, Aydoğdu M et al. Hyperhomocysteinemia prevalence among patients with venous thromboembolism. Clin Appl Thromb Hemost 2011;17:487-93.

5 Cattaneo M. Hyperhomocysteinemia and venous thromboembolism. Semin Thromb Hemost 2006;32:716-23. 